\title{
Pediatric central venous access devices: nursing interventions
}

\author{
Elizabeth A Duffy \\ Kathryn N Nelson \\ Department of Health Behavior and \\ Biological Sciences, The University \\ of Michigan School of Nursing, Ann \\ Arbor, MI, USA
}

This article was published in the following Dove Press journal:

Nursing: Research and Reviews

19 May 2017

Number of times this article has been viewed

\begin{abstract}
A central venous catheter (CVC) is an indwelling catheter that provides permanent or temporary stable venous access for both acute and chronically ill pediatric patients. These catheters provide stable venous access that can be used for a variety of medical purposes including drawing blood, hemodynamic monitoring, infusion of intravenous medications, infusion of intravenous fluids, chemotherapy, blood products, and parenteral nutrition. Each day, nurses access and care for CVCs in infants, children, and adolescents; the precision of this care can prevent life-threatening complications. The purpose of this review and the case study is to highlight the importance and components of evidence-based nursing practice in pediatric CVC care. A historical perspective of CVC care is provided in conjunction with current national initiatives to improve patient outcomes for children with CVCs. Infection prevention, clinical practice guidelines, quality improvement, and evidence-based care bundles are discussed.
\end{abstract}

Keywords: pediatric nursing, central venous catheters, central line-associated bloodstream infection, care bundles, pediatric case study

\section{Case study}

Ryker is a previously healthy 9-year old boy. He was brought to the emergency department by ambulance after being hit by a car while riding his bicycle. His injuries included fractures of the pelvis and femur, a pulmonary laceration, and a laceration of spleen. He required multiple surgeries to manage his injuries. During his second surgery, a right subclavian central venous catheter (CVC) was placed. This catheter provides stable venous access for administration of fluids, medications, and labs during his recovery. On hospital day \#10, Ryker developed a fever, and labs and blood cultures were drawn. His labs were significant for a white blood cell count of 22,000 cells $/ \mathrm{mm}^{3}$. The initial gram stain of his blood revealed gram-positive cocci in clusters with a culture positive for coagulase-negative staphylococcus. Ryker was started on antibiotics and his CVC was removed. He continued to recover from his injuries, and he responded well to treatment with antibiotics for his bloodstream infection. He was discharged to an inpatient rehabilitation program on hospital day \#18.

After further review, and ruling out other potential sources of infection such as a urinary tract infection, surgical site infections, and pulmonary infections, Ryker's positive bloodstream infection was classified as a central line associated-bloodstream infection (CLABSI) using the Center for Disease Control and Prevention (CDC) criteria. This health care-associated infection (HAI) triggered a review by the health care team. Physicians, nurses, respiratory therapists, pharmacists, and other interdisciplinary staff
Department of Health Behavior and Biological Sciences, The University of Michigan School of Nursing, 426 North Ingalls, Room 4I 34, Ann Arbor, MI 48109, USA

Tel +l 7347647879

$\mathrm{Fax}+\mathrm{I} 7349365525$

Email eaduffy@med.umich.edu
Nursing: Research and Reviews 2017:7 5I-56 
who cared for Ryker gathered to review the care provided and discuss strategies to prevent similar infections in the future. During the review, they estimated that CLABSI prolonged his hospitalization by at least 3 days. This is a hypothetical case study that is not based on one single patient.

\section{Benefits of central lines}

A CVC is an indwelling catheter that provides temporary or long-term stable venous access for both acute and chronically ill patients. These catheters provide stable venous access that can be used for a variety of medical purposes including drawing blood, hemodynamic monitoring, infusion of intravenous medication, infusion of intravenous fluids, chemotherapy, blood products, and parenteral nutrition. For some pediatric patients with acute and chronic illness, CVCs are an important tool in their medical care when temporary peripheral access is inadequate. There are multiple types of pediatric central lines and the decision regarding the type of line that is chosen for insertion is dependent upon the reason for the CVC, patient's diagnosis, age of the patient, patient weight, and time frame the CVC will be in place. Types of central lines include external catheters such as Hickman or Broviac, totally implanted catheters such as Mediports, and peripherally inserted central catheters.

\section{Risks associated with CVCs}

The first CVC was inserted in the 1920s. ${ }^{1}$ A CVC is a tunneled catheter that is surgically inserted into a central vein usually in the neck, upper chest, or groin and then passed under the skin. ${ }^{2}$ Mechanical complications such as air embolisms, catheter leaks, and hub separations were common complications in the early literature; however, these complications are now rare during central line placement. Central line-associated bloodstream infection was recognized as the most serious risk factor in the use of the CVC in the 1950 s and it continues to be a significant complication today. ${ }^{3}$

Today, it is widely recognized that complications associated with the use of a CVC contribute to morbidity and mortality and lead to increased health care utilization and cost; risks and benefits of CVC must be weighed carefully. Risks of CVCs include infection, malposition, pneumothorax, thrombus, embolism, tamponade, bleeding, arrhythmias, and catheter occlusion. ${ }^{4}$ One of the leading complications of central lines in pediatric patients is CLABSI with $10 \%$ of pediatric patients with a CVC experiencing a CLABSI at a rate of 1.63 CLABSIs per 1,000 catheter days. ${ }^{5}$ Decades after CLABSI was recognized as a significant complication associated with the use of CVCs, the prevention of CLABSI remains a high priority.

\section{Central line-associated bloodstream infection}

Infection prevention is a hallmark of nursing care. It is estimated that 722,000 HAIs occurred in acute-care facilities in 2011, resulting in 75,000 deaths. ${ }^{6}$ CLABSI is considered a HAI.

CLABSI is a preventable complication. A CLABSI is defined as a confirmed bloodstream infection in a patient with a central line in place within the 48-hour period before the onset of the bloodstream infection that is not secondary to an infection at another body site meeting the CDC National Healthcare Safety Network Criteria. ${ }^{7}$ According to this criterion, at least two or more blood cultures drawn on separate occasions should be positive from the same organism. CLABSI remains a significant source of mortality, morbidity, and additional medical expense for pediatric and adult patients each year..$^{8-11}$ Approximately 80,000 CLABSIs occur each year in hospitals, and 30,000 of those result in death. The cost of CLABSI to the United States health system is more than $\$ 2$ billion annually, with an average cost per patient of $\$ 45,000 .^{12}$

Each day nurses access and care for infants, children, and adolescents with CVCs; it is the precision of this care that prevents CLABSI. It is imperative that nurses are aware of evidence-based guidelines for CVC care to prevent infection. The purpose of this review is to highlight the importance and components of evidence-based nursing practice in $\mathrm{CVC}$ care.

\section{Collaborative efforts to prevent CLABSI}

Central line-associated bloodstream infection was once thought to be an often unavoidable complication of central lines. More recently, individual units and hospitals, as well as large quality improvement collaboratives, have demonstrated that consistent and meticulous care can drastically reduce the incidence of CLABSI. Prevention of CLABSI has been identified as a national priority by the Joint Commission through the national patient safety goals requiring hospitals to reduce the risk of health care-associated conditions. ${ }^{13,14}$ Government bodies, professional societies, health care organizations, and individuals throughout the world are focusing attention on the preventability of CLABSIs by implementing evidencebased practices outlined in the scientific literature. ${ }^{15}$ Over the past 15 years, multisite collaborative quality improvement initiatives have demonstrated statistically and clinically significant results in the prevention of CLABSI. ${ }^{12}$ Clinical practice guidelines (CPGs) have been developed based upon the results of this collaboration. 
Clinical practice guidelines are patient care recommendations based on the best available research evidence and practice experience. The National Academy for Medicine defines CPGs as statements that include recommendations intended to optimize patient care that are informed by a systematic review of evidence and an assessment of the benefits and harms of alternative care options. ${ }^{16}$ The generation of CPGs that provide recommended practices in the care of the CVC has resulted from the collaborative approach to prevent CLABSI. Several countries, regions, and organizations have established CPGs pertaining to the prevention of CLABSIs, authored by governmental, professional, and public health organizations. ${ }^{15}$

\section{Utilization of care bundles in quality improvement}

In order to prevent infection, health care organizations continue to implement CPGs. An inherent challenge in this implementation is the complexity and length of many CPGs. In order to simplify and ease the translation of evidence directly into practice, targeted care bundles have been developed.

A care bundle is a group of evidence-based preventative practices that when consistently implemented together improve the quality of patient care and achieve a better outcome than when implemented alone. Care bundles have been developed for the prevention of CLABSI. The CDC CLABSI prevention guidelines provide the basis for both an insertion bundle and a CVC maintenance care bundle. Central venous catheter insertion and daily maintenance care bundles have been used to reduce CLABSI in pediatric intensive care units, neonatal intensive care units, pediatric cardiothoracic units, pediatric stem cell transplant recipients, and inpatient pediatric oncology patients. ${ }^{17,18} \mathrm{Central}$ venous catheter insertion bundles focus on care practices during insertion of the $\mathrm{CVC}$, and maintenance care bundles address specific procedures for access, dressing changes, intravenous line care, and patient hygiene.

Nurses assume frontline responsibility for safe, quality CVC care to minimize the risk of patients contracting life-threatening infections. ${ }^{19}$ Implementation of bundled guidelines for the care of the CVC is consistent with Institute for Health Improvement's initiative to minimize practice variability. ${ }^{20}$ CLABSI can be reduced by $50 \%$ when caregivers use evidence-based CVC bundles, observation checklists, and marketing and education strategies. ${ }^{21}$

\section{Bundle components}

Bundle components include the minimal standards for the care of the CVC. Institutional guidelines consistent with the
CDC provide the framework for each care bundle. ${ }^{7}$ Central venous catheters bundles vary between institutions but often provide guidance on insertion practices, care of the central line once it is in place, and guidelines for health care teams.

One theme throughout bundle guidelines is the importance of hand hygiene and maintenance of clean and antiseptic techniques. In order to prevent CLABSIs, it is important to understand the presumed routes of transmission of infections, which include extraluminal transmission and intraluminal transmission. Extraluminal transmission involves the transmission of microorganisms from the patient's skin along the catheter resulting in colonization of the catheter tip. Intraluminal transmission involves direct contamination of the catheter or tubing, seeding of the catheter from another site of infection (urinary tract infection, pneumonia, etc.), or infusion of contaminated medication or fluids. ${ }^{22}$ Bundle components generally target extraluminal transmission and intraluminal transmission via direct contamination of the catheter or tubing.

Nurses, especially in the intensive care units (ICU) and operating room settings, are often involved during the placement of central lines. Even if they are not performing the insertion, nurses play an important role in monitoring and supporting insertion practices. CVC insertion bundle practices often include the following elements: ${ }^{23}$

- Hand hygiene prior to insertion: Proper hand hygiene is required before and after palpating catheter insertion sites as well as before and after inserting, replacing, accessing, repairing, or dressing an intravascular catheter. Since 1977, at least seven prospective studies have shown that improvement in hand hygiene significantly decreases a variety of infectious complications. Central venous catheters carry a substantially greater risk for infection. ${ }^{24-29}$

- Appropriate skin antisepsis: The clean skin is disinfected with an appropriate antiseptic before catheter insertion and during dressing changes. In a study from 1991, preparation of central venous and arterial sites with a $2 \%$ aqueous chlorhexidine gluconate lowered bloodstream infection rates compared with site preparation with $10 \%$ povidone-iodine or $70 \%$ alcohol. A meta-analysis from 2002 demonstrated that the use of a chlorhexidine-containing preparation decreased central catheter-related infections by $49 \%$ relative to povidoneiodine preparations. ${ }^{24,30-32}$

- Maximal sterile barrier precautions include sterile gloves, sterile gown, cap, mask and sterile full bed drape: The use of sterile barrier precautions during the insertion of CVCs substantially reduces the incidence of CLABSI 
compared with standard precautions (eg, sterile gloves and small drapes). ${ }^{24,33-36}$

- Avoidance of routine replacement of functioning CVCs as a strategy to prevent CLABSI: An effective strategy for preventing CLABSI is to eliminate or reduce exposure to CVCs. The decision regarding the need for a catheter is complex, however, and difficult to standardize into a practice guideline.

- The multidisciplinary team should systematically evaluate daily whether a CVC is necessary and potentially could be removed. . $^{2,37,38}$

Once the line is in place, most daily care of the CVC is performed by the nurse. CVC daily maintenance care bundles typically include the following practices: ${ }^{23}$

- Hand hygiene prior to CVC care. ${ }^{24-29}$

- Sanitize the access port with alcohol, chlorhexidine/ alcohol, povidone-iodine, or iodophors before and after each use according to institutional policy. This is often called "Scrub the Hub". 24,39-41

- Minimize the number of times the CVC is accessed. ${ }^{24,39-41}$

- Change intravenous tubing and caps according to institutional time frames and type of infusion. Intravenous tubing and cap change is also dependent upon the weight of the patient, underlying diagnosis, and type of infusion. ${ }^{24,39-41}$

- Assess blood return from the CVC every 24 hours with the use of anti-thrombolytics as determined by institutional guidelines if blood return is absent.

- Central venous catheters dressings must remain clean, dry, and intact compliant with institutional guidelines regarding types of dressings, frequency of dressing changes, and protocols for dressing changes. In general, gauze and tape dressing are changed every 2 days and transparent dressings are changed every 7 days or when the dressing becomes damp, loose, or soiled. ${ }^{24,42,43}$

- Daily hygiene, linen change, and activities of daily life that are consistent with infection prevention.

- Prevention of CLABSI requires the active engagement of the entire health care team. Team practices that can help prevent CLABSI include: Central venous catheters should be removed as soon as possible and the necessity of the CVC should be evaluated daily. ${ }^{23,24,37,38}$

- Documentation of CVC care in the nursing notes and patient flow sheets verify that all bundle components have been completed and that daily CVC care is compliant with the evidence-based care bundle. The overarching goal in the utilization of care bundles in CVC care is that all components are completed each time; the absence of one element predisposes the patient to CLABSI. Standardization of nursing documentation can facilitate process improvement in the documentation of CVC care bundles. $^{21}$

\section{Importance of nursing care to decrease CLABSI}

In 2006, a major national initiative was conducted by the National Association of Children's Hospitals and Related Institutions that focused on CLABSI among pediatric critically ill patients; this initiative reported a reduction in CLABSI from 6.3 infections per 1,000 catheter days to 4.3 infections per 1,000 catheter days. ${ }^{44}$ The importance of nursing practice in relation to the performance of maintenance care was highlighted. It was noted that maintenance care practices are complex and multidimensional for nurses to master reliably. Maintenance care needs to be consistently performed with precision many times a day for each patient. ${ }^{18}$ Furthermore for children in ICUs, the main driver for CLABSI rate reduction is the daily central line maintenance delivered primarily by nurses. ${ }^{45}$

\section{Strategies to increase compliance with bundle elements}

Nurses have the most direct, ongoing role in the care of patients and the interventions or procedures that put patients at risk of infection. ${ }^{46}$ The engagement of nursing champions to implement and sustain CVC care bundles is essential. Nursing champions that recognize the importance of care bundles and the evidence that provides the framework for the bundle elements facilitates buy-in from other nursing staff. Nursing staff education that incorporates unit specific CLABSI rates also improves bundle compliance. The educational methods chosen should take into consideration the preferred methods of learning, principles of adult education, resources available, cultural norms, and languages spoken by health care personnel. ${ }^{15,47,48}$

Engagement of the patient and family in CVC care bundles is imperative. Central venous catheter line rounds facilitated by nursing champions can be an important tool in CLABSI prevention. Verbal discussion between the patient, family, and nursing staff in conjunction with a chart review provides valuable information regarding bundle compliance that facilitates targeted education. In addition, physician inquiry into use and care of the CVC during interdisciplinary rounds strengthens the importance of daily CVC care and reinforces education provided by nursing staff. 


\section{CLABSI follow-up}

In conjunction with the work surrounding CLABSI prevention, nurses also need to respond when a patient develops a CLABSI. Root Cause Analysis (RCA), first developed to analyze industrial accidents, is a structured analysis now commonly used to analyze adverse outcomes in health care. The RCA process does not focus on errors of any single individual but instead uses a systems approach to engage the health care team to identify how and why the event occurred and risks for similar circumstances in the future. Root cause analysis is an effective tool to assist a health care team that is fully engaged in quality improvement interventions. ${ }^{49}$ When combined with CVC insertion and maintenance bundles, RCA assists in identifying additional processes and systems that may contribute to CLABSIs and additional areas of improvement. ${ }^{50}$ Nurses are uniquely positioned to participate in the process of RCA due to their frontline involvement of CVC care.

\section{Conclusion}

Health care-associated infections are considered the most frequent adverse events in health care delivery. ${ }^{24}$ Decreasing variance in practice through the consistent use of evidencebased care bundles in concert with interdisciplinary collaboration is foundational for decreasing CLABSI. According to the Agency for Healthcare Research and Quality, there has been a 17\% decline in HAIs between 2010 and 2014, which resulted in 2.1 million fewer incidents of harm during that period. ${ }^{24}$ Nurses are uniquely positioned in the health care team to be able to reduce rates of CLABSI. Nurses can prevent CLABSIs by providing meticulous CVC care to their patients, speaking up if they identify care practices that place patients at risk for CLABSI, and by participating in interdisciplinary groups to prevent CLABSI. As evidencebased guidelines for care and maintenance of CVCs continue to evolve, it is important for nurses to be active in ongoing CLABSI research and for nurses to work to align practices and policies with the latest evidence-based recommendations. Implementation of the most current evidence-based guidelines for CLABSI prevention will result in fewer patients like Ryker, that experience additional morbidity or mortality as a result of a HAI.

\section{Disclosure}

The authors report no conflicts of interest in this work.

\section{References}

1. Zingg W, Cartier-Fässler V, Walder B. Central venous catheter-associated infections. Best Pract Res Clin Anaesthesiol. 2008;22(3):407-421.
2. University of Michigan. Available from: http://surgery.med.umich.edu/ pediatric/patient/conditions/A-M/broviac_placement.shtml. Accessed November 7, 2016.

3. Raad I, Hanna H, Maki D. Intravascular catheter-related infections: advances in diagnosis, prevention, and management. Lancet Infect Dis. 2007;7(10):645-657.

4. de Jonge RC, Polderman KH, Gemke JB. Central venous catheter use in the pediatric patient: mechanical and infectious complications. Pediatr Crit Care Med. 2005;6(3):329-339.

5. Ullman AJ, Marsh N, Mihala G, Cooke M, Rickard CM. Complications of central venous access devices: a systematic review. Pediatrics. 2015;136(5):e1331-e1344.

6. Centers for Disease Control and Prevention. Available from: http:// www.cdc.gov/HAI/bsi/CLABSI-resources.html. Accessed November 7, 2016.

7. The Centers for Disease Control and Prevention. Device Assisted Module: Central Line-Associated Infection; 2016. Available from: http:// www.cdc.gov/nhsn/pdfs/pscmanual/4psc_clabscurrent.pdf. Accessed November 7, 2016.

8. Costello JM, Morrow DF, Graham DA, Potter-Bynoe G, Sandora TJ, Laussen PC. Systematic intervention to reduce central line-associated bloodstream infections rates in a pediatric cardiac intensive care unit. Pediatrics. 2008;121:915-923.

9. Harrigan S, Hurst D, Lee C, et al. Developing and implementing quality initiatives in the ICU: strategies and outcomes. Crit Care Nurs Clin North Am. 2006;18:469-479.

10. McCaskey MS. Transferring central line care evidence into practice on pediatric acute care units. J Pediatr Nurs. 2013;28(6):e57-e63.

11. Miller M, Griswold M, Harris J, et al. Decreasing PICU catheterassociated bloodstream infections: NACHRI's quality transformation efforts. Pediatrics. 2010;125:206-213.

12. Pronovost PJ, Marsteller JA, Goeschel CA. Preventing bloodstream infections: a measurable national success story in quality improvement. Health Aff (Millwood). 2011;30(4):628-634.

13. Steering Committee on Quality Improvement and Management and Committee on Hospital Care. Policy statement-principles of pediatric patient safety: reducing harm due to medical care. Pediatrics. 2011;127:1199-1210.

14. The Joint Commission. National patient safety goals. 2010. Available from: https://www.jointcommission.org/topics/clabsi_toolkit.aspx. Accessed January 5, 2017.

15. The Joint Commission. Preventing Central Line-Associated Bloodstream Infections: A Global Challenge, A Global Perspective. Available from: https://www.jointcommission.org/assets/1/18/CLABSI_Monograph.pdf. Accessed November 7, 2016.

16. Consensus report, Institute of Medicine. Clinical practice guidelines we can trust. Available from: http://nationalacademies.org $/ \mathrm{hmd} /$ reports/2011/clinical-practice-guidelines-we-can-trust.aspx. Accessed January 5, 2017.

17. Rinke ML, Chen AR, Bundy DG, et al. Implementation of a central line maintenance care bundle in hospitalized pediatric oncology patients. Pediatrics. 2012;130(4):e996-e1004.

18. Miller MR, Niedner MF, Huskins, WC, et al; National Association of Children's Hospitals and Related Institutions Pediatric Intensive Care Unit Central Line-Associated Bloodstream Infection Quality Transformation Teams. Reducing PICU central-line associated bloodstream infections: 3-year results. Pediatrics. 2011;128(5):e1077-e1083.

19. Secola RN, Lewis MA, Pike N, Needleman J, Doering L. "Targeting to Zero" in pediatric oncology: a review of central venous catheter-related bloodstream infections. J Pediatr Oncol Nurs. 2010;29(1):14-27.

20. Institute for Health care Improvement (IHI) Critical care. What is a bundle?; 2006. Available from: http://www.ihi.org/IHI/Topics/Critical Care/IntensiveCare/ImprovementStories/WhatIsaBundle.htm. Accessed November 7, 2016.

21. Brungs SM, Render ML. Using evidence-based practice to reduce central line infections. Clin J Oncol Nurs. 2006;10:723-725. 
22. The Joint Commission. Preventing Central Line-Associated Bloodstream Infections: A Global Challenge, A Global Perspective. Oak Brook, IL: Joint Commission Resources. Available from: http://www. PreventingCLABSIs.pdf. Accessed May 2012. Accessed November 7, 2016.

23. O' Grady NP, Alexander M, Burns L, et al; Healthcare Infection Control Practices Advisory Committee (HICPAC). Guidelines for the prevention of intravascular catheter-related infections. Clin Infect Dis. 2011;52(9):e162-e193.

24. Agency for Healthcare Research and Quality. Central Line-Associated Bloodstream Infections. Available from: http://www.ahrq.gov/profess ionals/education/curriculum-tools/clabsitools/clabsitoolsap2.html. Accessed November 7, 2016.

25. Pittet D, Hugonnet S, Harbarth S, et al. Effectiveness of a hospital-wide programme to improve compliance with hand hygiene. Infection Control Programme. Lancet. 2000;356(9238):1307-1312.

26. Larson EL. APIC guideline for handwashing and hand antisepsis in health care settings. Am J Infect Control. 1995;23(4):251-269.

27. Rosenthal VD, Guzman S, Safdar N. Reduction in nosocomial infection with improved hand hygiene in intensive care units of a tertiary care hospital in Argentina. Am J Infect Control. 2005;33(7):392-397.

28. Boyce JM, Pittet D; Healthcare Infection Control Practices Advisory Committee; HICPAC/SHEA/APIC/IDSA Hand Hygiene Task Force. Guideline for hand hygiene in health-care settings. Recommendations of the healthcare infection control practices advisory committee and the HICPAC/SHEA/APIC/IDSA Hand Hygiene Task Force. Society for Healthcare Epidemiology of America/Association for Professionals in Infection Control/Infectious Diseases Society of America. MMWR Recomm Rep. 2002;51(RR-16):1-45.

29. Yilmaz G, Koksal I, Aydin K, Caylan R, Sucu N, Aksoy F. Risk factors of catheter-related bloodstream infections in parenteral nutrition catheterization. JPEN J Parenter Enteral Nutr. 2007;31(4):284-287.

30. Maki DG, Ringer M, Alvarado CJ. Prospective randomised trial of povidone-iodine, alcohol, and chlorhexidine for prevention of infection associated with central venous and arterial catheters. Lancet. 1991;338(8763):339-343.

31. Chaiyakunapruk N, Veenstra DL, Lipsky BA, Saint S. Chlorhexidine compared with povidone-iodine solution for vascular catheter-site care: a meta-analysis. Ann Intern Med. 2002;136(11):792-801.

32. Humar A, Ostromecki A, Direnfeld J, et al. Prospective randomized trial of $10 \%$ povidone-iodine versus $0.5 \%$ tincture of chlorhexidine as cutaneous antisepsis for prevention of central venous catheter infection. Clin Infect Dis. 2000;31(4):1001-1007.

33. Mermel LA, McCormick RD, Springman SR, Maki DG. The pathogenesis and epidemiology of catheter-related infection with pulmonary artery Swan-Ganz catheters: a prospective study utilizing molecular subtyping. Am J Med. 1991;91(3B):197S-205S.

34. Raad II, Hohn DC, Gilbreath BJ, et al. Prevention of central venous catheter-related infections by using maximal sterile barrier precautions during insertion. Infect Control Hosp Epidemiol. 1994;15(4 Pt 1):231-238.

35. Hu KK, Lipsky BA, Veenstra DL, Saint S. Using maximal sterile barriers to prevent central venous catheter-related infection: a systematic evidence-based review. Am J Infect Control. 2004;32(3):142-146.
36. Young EM, Commiskey ML, Wilson SJ. Translating evidence into practice to prevent central venous catheter-associated bloodstream infections: a systems-based intervention. Am J Infect Control. 2006; 34(8):503-506.

37. Lederle FA, Parenti CM, Berskow LC, Ellingson KJ. The idle intravenous catheter. Ann Intern Med. 1992;116(9):737-738.

38. Parenti CM, Lederle FA, Impola CL, Peterson LR. Reduction of unnecessary intravenous catheter use. Internal medicine house staff participate in a successful quality improvement project. Arch Intern Med. 1994;154(16):1829-1832.

39. Coopersmith CM, Zack JE, Ward MR, et al. The impact of bedside behavior on catheter-related bacteremia in the intensive care unit. Arch Surg. 2004;139(2):131-136.

40. Coopersmith CM, Rebmann TL, Zack JE, et al. Effect of an education program on decreasing catheter-related bloodstream infections in the surgical intensive care unit. Crit Care Med. 2002;30:59-64.

41. Berenholtz SM, Pronovost PJ, Lipsett PA, et al. Eliminating catheterrelated bloodstream infections in the intensive care unit. Crit Care Med. 2004;32:2014-2020.

42. O'Grady NP, Alexander M, Dellinger EP, et al. Guidelines for the prevention of intravascular catheter-related infections. The Hospital Infection Control Practices Advisory Committee, Center for Disease Control and Prevention, U.S. Pediatrics. 2002 Nov;110(5):e51.

43. Newell-Stokes V, Broughton S, Giuliano KK, Stetler CB. Developing an evidence-based practice procedure: maintenance of central venous catheters. Clin Nurse Spec. 2001;15:199-204.

44. Jeffries HE, Mason W, Brewer M, et al. Prevention of central venous catheter-associated bloodstream infections in pediatric intensive care units: a performance improvement collaborative. Infect Control Hosp Epidemiol. 2009;30:645-651.

45. Nowak JE, Brilli RJ, Lake MR, et al. Reducing catheter-associated bloodstream infections in the pediatric intensive care unit: business case for quality improvement. Pediatr Crit Care Med. 2010;11(5): 579-587.

46. Kübler A, Duszynska W, Rosenthal VD, et al. Device-associated infection rates and extra length of stay in an intensive care unit of a university hospital in Wroclaw, Poland: International Nosocomial Infection Control Consortium's (INICC) findings. J Crit Care. 2012;27(1):105.e5-e10.

47. Pérez Parra A, Cruz Menárguez M, Pérez Granda MJ, Tomey MJ, Padilla B, Bouza E. A simple educational intervention to decrease incidence of central line-associated bloodstream infection (CLABSI) in intensive care units with low baseline incidence of CLABSI. Infect Control Hosp Epidemiol. 2010;31(9):964-947.

48. Pronovost P, Needham D, Berenholtz S, et al. An intervention to decrease catheter-related bloodstream infections in the ICU. $N$ Engl $J$ Med. 2006;355(26):2725-2732.

49. Exline MC, Ali, NA, Zikri N, et al. Beyond the bundle-journey of a tertiary care medical intensive care unit to zero central line-associated bloodstream infections. Crit Care. 2013;17(2):R41.

50. Walz JM, Ellison RT 3rd, Mack DA, et al; CCOC Research Group. The bundle "plus": the effect of a multidisciplinary team approach to eradicate central line-associated bloodstream infections. Anesth Analg. 2015;120(4):868-876.
Nursing: Research and Reviews

\section{Publish your work in this journal}

Nursing: Research and Reviews is an international, peer-reviewed, open access journal publishing original research, reports, reviews and commentaries on all aspects of nursing and patient care. These include patient education and counseling, ethics, management and organizational issues, diagnostics and prescribing, health outcomes, economics and

\section{Dovepress}

resource management, improving patient safety in all settings. The manuscript management system is completely online and includes a very quick and fair peer-review system. Visit http://www.dovepress. com/testimonials.php to read real quotes from published authors. 